\title{
Adipose Tissue-Specialized Immunologic Features Might Be the Potential Therapeutic Target of Prospective Medicines for Obesity
}

\author{
Fan Yao, ${ }^{1}$ Ming Zhang, ${ }^{1}$ and Li Chen ${ }^{1,2}$ \\ ${ }^{1}$ Department of Pharmacology, College of Basic Medical Sciences, Jilin University, Changchun 130021, China \\ ${ }^{2}$ School of Nursing, Jilin University, Changchun 130021, China \\ Correspondence should be addressed to Li Chen; chen_lab@163.com
}

Received 4 January 2017; Revised 3 March 2017; Accepted 13 March 2017; Published 30 March 2017

Academic Editor: Aimin Xu

Copyright ( 2017 Fan Yao et al. This is an open access article distributed under the Creative Commons Attribution License, which permits unrestricted use, distribution, and reproduction in any medium, provided the original work is properly cited.

\begin{abstract}
Excessive lipid accumulation in adipose tissue is either the source of obesity or the cause and result of chronic local inflammation, and recent studies indicate that the accumulation may induce many other specialized immunologic features with macrophages and epidemic diseases. We analyze the effective stages of immune cells in adipose tissue, including macrophage recruitment, macrophage polarization, and macrophage-like phenotype preadipocyte possession to find optimal sites as drug targets. Subsequently, some main signaling pathways are summarized in this review, including the AMP-activated protein kinase (AMPK) pathway, the JNK signaling pathway, and a novel one, the Notch signaling pathway. We illustrate all these points in order to determine the general pathogenesis of chronic low-grade local inflammation in adipose tissue and the related signaling pathways. In addition, signal-associated prospective compounds, such as berberine, are summarized and discussed with potential targets in pathogenesis. This might provide some possible thoughts and novel therapies for studying chronic inflammatory diseases, such as insulin resistance and type 2 diabetes mellitus.
\end{abstract}

\section{Introduction}

The World Health Organization (WHO) reported that more than 1.4 billion adults and older individuals were overweight in 2012. More than 200 million men and approximately 300 million women were classified as obese. Additionally, more than 40 million children under the age of 5 were classified as being obese in 2012. As a worldwide and dangerous epidemic, obesity increases the risk of some chronic diseases and induces low-grade inflammation with many other complications, such as atherosclerosis, cardiovascular problems, insulin resistance, and type 2 diabetes mellitus [1-5]. Adipose tissue also plays a crucial role in the generation of low-grade inflammation and progression. In hypertrophic adipose tissue, many cytokines and chemokines are important contributors in different regulatory pathways, especially cytokines that are activated and released by macrophages/ monocytes immune cells. In this review, we will summarize the relevant reports and research about chronic low-grade inflammation in adipose tissue to find prospective medicines. The process contains the chronic local inflammation mechanism of immune cells in adipose tissue, such as macrophage recruitment, macrophage polarization, and macrophage-like phenotype preadipocyte. Subsequently, pertinent signaling pathways will be illustrated and generalized involving the adenosine monophosphate-activated protein kinase (AMPK) signaling pathway and the Notch pathway for potential medical targets.

\section{Origin of Macrophages/Monocytes in Chronic Inflammation of Local Adipose Tissue}

In the foetal stage, the yolk sac and foetal liver haematopoiesis are the embryonic precursors for macrophages $[6,7]$. Most of the tissue-resident macrophages were thought to arise from embryonic precursors $[8,9]$. However, a novel method has helped illuminate the fact that the sole origin of tissue-resident macrophages in the liver and pancreas might be yolk sac macrophages. This leads to the origin of tissueresident macrophages being a controversial topic $[7,10]$. In the postnatal stage, blood monocytes developed from haematopoietic stem cells in the bone marrow are the main 
precursors of macrophages in the circulation system [11-13]. Similarly, T cells promote monocyte differentiation and macrophage polarization [14]. These should be the classical approach for macrophage production. However, currently, researchers have found that macrophages/monocytes have been included in the adipose tissue for regulation of chronic local inflammation. Although the mechanism of chronic inflammation has not been clearly elucidated, the possible modulating methods and pathways have been reported and discussed. The adipose tissue contains adipocytes, preadipocytes, stem cells, and other stromal cells; macrophages as immune cells and homeostatic regulators make up almost $10 \%$ of the normal adipose tissue. Macrophages from normal adipose tissue take the function of anti-inflammation to maintain homeostasis. In contrast, when the homeostasis is broken up, as in obesity, the number of macrophages will increase significantly, contributing up to $50 \%$ of the cells in adipose tissue. These cells contain $85 \%$ of macrophages from recruitment and infiltration as well as $15 \%$ from preadipocyte macrophage-like phenotypes [15-17]. Any proposed medicine should play a key role in modulating the large amount of macrophages in adipose tissue for disease treatments.

2.1. Macrophage Recruitment and Infiltration. Under dietinduced obesity conditions, the activation of macrophages results in chronic low-grade inflammation in local adipose tissue. This could be induced by macrophage recruitment. Lumeng et al. [18] reported numerous F4/80 ${ }^{+} \mathrm{CD} 16 / 32^{+}$

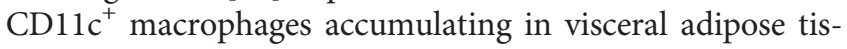
sue. Another important difference is the higher expression of proinflammatory factors such as tumour necrosis factor alpha (TNF- $\alpha$ ), interleukin- $1 \beta$ (IL-1 $\beta$ ), and inducible nitric oxide synthase (iNOS). The exact mechanism remains a topic of study. It is already understood that the activated monocytes appear to accumulate in local tissue that is stimulated via cytokines such as MCP-1 secreted by macrophages. The recruitment and infiltration of monocytes/macrophages might be associated with adipocyte hypertrophy, local hypoxia, and the interaction between adipocytes and macrophages [19]. Moreover, MCP-1 is a type of inflammationrelated genetic code cytokines that plays a critical role in the pathways of macrophage recruitment and the regulation of insulin sensitivity. Previous studies of obese processes illustrated that increased adipocyte volume could induce angiogenesis and increase adipose tissue consumption. When the rate of increased adipocyte volume is not equal to the rate of vascular proliferation, lipids excessively accumulate in adipocytes. Subsequently, anoxia tissue leads to a volume increase in adipocytes as well as cell apoptosis. Then, excessive amounts of saturated fatty acid and accumulated cytokines are released [20]. In detail, the regulating pathways might be highly associated with the proteins peroxisome proliferator-activated receptor $\gamma(\operatorname{PPAR} \gamma)$, nuclear factor$\kappa \mathrm{B}(\mathrm{NF}-\kappa \mathrm{B})$, and MCP-1.

2.2. Macrophage Polarization. The macrophages in chronic inflammation could also come from macrophage polarization. This could be regulated by many factors. Based on the phenotype and a variety of secreted cytokines, there are two types of macrophages, classically activated M1 and alternatively activated M2. It is important to note that M1 has the function of proinflammation by secreting cytokines such as TNF- $\alpha$, IL- $1 \beta$, iNOS, and MCP-1 [21]. M2 takes on the function of anti-inflammation and tissue regeneration. With the help of IL-4, Arg-1, IL-10, and TGF- $\beta$, macrophages might be induced to polarize M2 and secrete cytokines such as TGF- $\beta$, VEGF, and EGF or additional Arg- 1 and IL-10. In the final stage of inflammation, M2 enhances tissue repair and fibrosis [22, 23]. Additionally, two crucial nuclear factors, PPAR $\gamma$ and NF- $\kappa \mathrm{B}$, are also the key targets in the modulation of macrophage polarization. Furthermore, PPAR $\gamma$ has been proven to be the important factor of M2 generation. It activates M2 polarization with the function of antiinflammation. If PPAR $\gamma$ has not been activated, the alternatively activated pathway will be blocked [24, 25]. Meanwhile, PPAR $\gamma$ blocked the proinflammatory pathway of NF- $\kappa \mathrm{B}$ and inhibited the expression of relative factors such as TNF- $\alpha$ [26]. Therefore, macrophage polarization is one of the important processes in chronic inflammation. Moreover, in a diet-induced obese mouse model, macrophage polarization can be regulated for influencing obesity-induced adipose tissue inflammation or insulin resistance. This might occur in interferon tau therapy, in chronic Trypanosoma cruzi infection, or with miR-130b assistance. This procession is also correlated with PPAR $\gamma$ [27-29]. Therefore, in local adipose tissue, macrophage polarization plays an important role in local chronic inflammation and insulin resistance.

2.3. Macrophage-Like Phenotype Preadipocytes. Macrophagelike phenotype preadipocytes are thought to originate from undifferentiated white adipocytes. The lineage tracing of adipose progenitors are generalized first. Adipocytes contain white adipocytes, brown adipocytes, and beige adipocytes that resemble white adipocytes but have the same function of classical brown adipocytes [30]. All of them originate from mesenchymal precursor cells. The majority of white adipocytes derive from $\mathrm{Myf5}^{-}$precursors and some white adipocyte precursors originate from the $\mathrm{Myf}^{+}$lineage. Mature white adipocytes derive from white adipocyte precursors or named preadipocytes in adulthood [31-34].

Based on previous studies, a question has developed about the relationship between adipocytes and macrophages. First, the major mediators of inflammation, for example, ILs and TNF- $\alpha$, have been found to be secreted from adipocytes and macrophages. Additionally, leptin takes part in not only proinflammation but also in energy metabolism, in particular, through $\mathrm{T}$ lymphocyte proliferation and macrophage activity [35]. In the recent years, the new definition of cell reprogramming has become an increasingly popular concept in advanced fields. Except for macrophage recruitment and polarization, other immune cells for local inflammation of adipose tissue may come from macrophage-like phenotype preadipocytes; we are working to identify these macrophagelike type preadipocytes and explore their functions. This kind of possible reprogramming means not only stem cell proliferation and differentiation but also genetic expression reprogramming that is induced by stimuli and changes in cell phenotype or function under certain conditions [36]. Adipose 
tissue is the potential area to generate cell reprogramming. For preadipocytes, they could be differentiated from adipose stem cells. Under chronic inflammation conditions, preadipocytes result in local inflammation caused by cytokines and might appear as macrophage phenotypes. The preadipocytes are stopped from differentiating into adipocytes [37]. Moreover, some findings confirm that there is an essential nuclear factor, $\operatorname{PPAR} \gamma$, that mediates the process of preadipocytes differentiating into adipocytes.

\section{Related Signaling Pathways}

In addition to immune cells studies in chronic local inflammation mechanism, several related signaling pathways have been found and explored. These pathways include the adenosine monophosphate-activated protein kinase (AMPK) signaling pathway, the C-Jun N-terminal kinase (JNK) signaling pathway, the Notch signaling pathway, the PI3K/Akt signaling pathway, and the JAK/STAT signaling pathway, which have been studied in the last few decades. The first three signaling pathways will be generalized below; they are linked with the chronic low-grade inflammation mechanism. In addition, the key signals might become the targets of prospective drugs in further studies.

\subsection{Adenosine Monophosphate-Activated Protein Kinase} (AMPK) Signaling Pathway. AMPK is a critical regulator in energy metabolism at the molecular level, in particular in glucose metabolism [38-40]. AMPK in the hypothalamus is a common regulator of weight gain. As a stress sensor, AMPK can be activated by a cluster of factors such as oxidative, metabolic, and physical stresses [39, 41, 42]. According to the heterotrimeric complex with one catalytic $\alpha$ subunit and two regulatory units $\beta$ and $\gamma$, AMPK contains two isoforms of $\alpha(\alpha 1$ and $\alpha 2)$ and $\beta$ ( $\beta 1$ and $\beta 2)$ with three $\gamma$ subunits $(\gamma 1, \gamma 2$, and $\gamma 3)$. The distinctive structure of AMPK isoforms is expressed differently in mammalian tissues [43]. Furthermore, AMPK is a key modulator in type 2 diabetes that is related to insulin sensitivity. This may also be mainly associated with obesity-induced inflammation and insulin sensitivity. Additionally, in adipocytes, the accumulation of lipids, together with the ectopic storage of fat in the pancreas, muscles, and other internal organs, may stimulate immune system defense and could provoke proinflammatory cytokine secretion and macrophages/monocytes recruitment, especially in adipose tissue [43-45]. With the help of several pharmacological activators or adipokines, AMPK inhibited the inflammatory reaction. For instance, the inflammatory response induced by lipopolysaccharide (LPS) was inhibited by activating AMPK with 5 -aminoimidazole-4-carboxamide riboside (AICAR), a classical activator of AMPK $[43,46]$. Another example for consideration is the findings of Steinberg et al. [47] who demonstrated that tumour necrosis factor- $\alpha$ (TNF- $\alpha$ ) might suppress AMPK activity by aggravating the expression of protein phosphatase $2 \mathrm{C}$, an inhibitor of the AMPK signaling pathway.

In the AMPK signaling pathway, AMPK inhibited NF- $\kappa \mathrm{B}$ p65 phosphorylation, suppressed gene expression of proinflammatory adipocytokines, and upregulated PPAR $\gamma$ expression [48]. Specifically, AMPK indirectly inhibited NF- $\kappa$ B signaling through some downstream mediators, such as silent information regulator 1 (SIRT1) and peroxisome proliferator-activated receptor $\gamma$ coactivator $1 \alpha$ (PGC- $1 \alpha)$ [43]. In the AMPK-SIRT1-NF- $\kappa B$ signaling pathway, there is a feedback loop during the energy deficiency. SIRT1 deacetylase was activated by AMPK via increasing cellular $\mathrm{NAD}^{+}$ levels. In contrast, AMPK was activated by LKB1 activity that is stimulated by SIRT1 $[49,50]$. Additionally, the acetylation of NF- $\kappa \mathrm{B}$ p65 improved its transactivation capacity. Conversely, SIRT1 interacted with p65 and finally deacetylated the p65 protein at lysine 310 . Thus, AMPK inhibited NF$\kappa B$ signaling via SIRT1-induced deacetylation $[43,51]$. Similarly, fatty acids are involved in the AMPK-PGC- $1 \alpha / \mathrm{NF}-\kappa \mathrm{B}$ signaling pathway, inducing the association of PGC- $1 \alpha$ factor with NF- $\kappa$ B p50 in hepatocytes. Moreover, PGC- $1 \alpha$ and p50 might bind to the IL-10 promoter and lead to the expression of IL-10 cytokine [52], because NF- $\kappa \mathrm{B}$ is a key mediator in fat-induced inflammation. Both of these indirect regulations in adipocytes by AMPK demonstrated that the AMPK signaling pathways could be crucial mediation pathways in adipose tissue chronic inflammation (Figure 1).

3.2. C-Jun N-Terminal Kinase (JNK) Signaling Pathway. The JNK signaling pathway belongs to the superfamily of mitogen-activated protein kinases (MAPKs) and is a major modulator of cell proliferation, differentiation and apoptosis [53-55]. There are three different isoforms, JNK1, JNK2, and JNK3, in the JNKs family; however, only the Jnk1 and Jnk2 genes can be expressed ubiquitously in all tissues [56, 57]. Related JNK proteins are expressed ubiquitously in most tissues [55]. Among these tissues, JNK in macrophages plays a significant role in the establishment of fat-induced insulin resistance and chronic inflammation, as well as macrophages accumulation and proinflammatory macrophage polarization $[18,58]$.

Furthermore, in the JNK signaling pathway, JNK1/2 was one of the indirect mediators for the process in which TNF- $\alpha$ decreased PPAR $\gamma$ and glucose transporter isoform 4 (GLUT4) expressions in adipocytes [59]. Namely, TNF- $\alpha$ maintained prolonged activation of JNK1/2 via TNF- $\alpha$ receptor 1 (TNFR1) and phosphorylates JNK downstream transcription factor c-Jun that restrained Map4k4 mRNA expression. In addition, Map4k4 expression aggravated the inhibition of PPAR $\gamma$, while TNF- $\alpha$ activated NF- $\kappa$ B by phosphorylation of JNK1/2 and p38 [59]. These two modulators are associated with adipose tissue inflammation and insulin resistance. Therefore, the JNK pathway could also be a useful signaling pathway in the treatment of obesity-induced syndromes and diseases (Figure 1).

3.3. Notch Signaling Pathway. The Notch signaling pathway could maintain tissue renewal by promoting or inhibiting cell proliferation, cell differentiation, and cell death [60]. The Notch family has four transmembrane Notch receptors expressed in mammals that may combine with the Notch signal and regulate distinct downstream factors [55]. Some experiments have shown that pharmacological suppression of Notch signaling in obese mice augmented Ucp1 expression, reduced blood glucose, and ameliorated obesity [61]. 


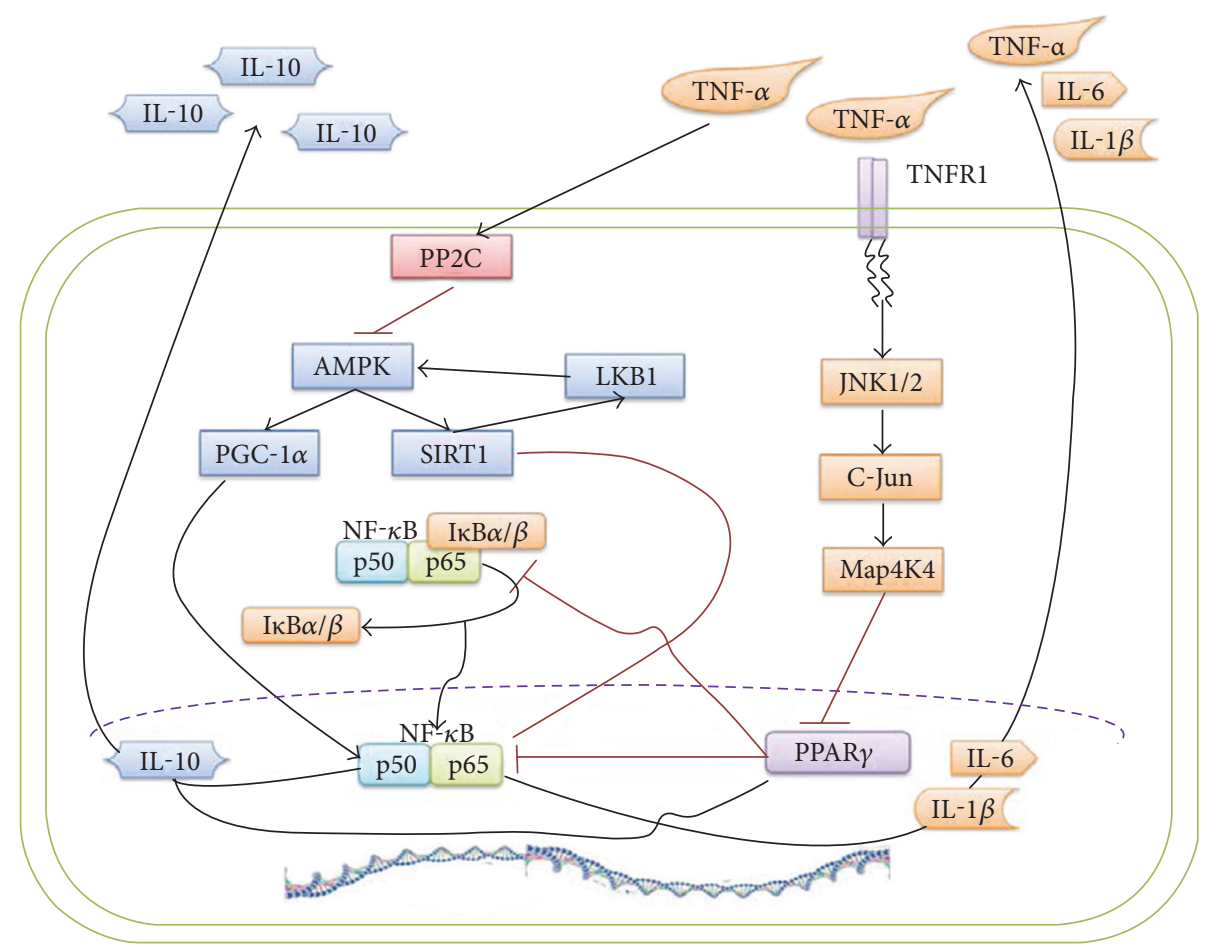

FIGURE 1: The AMPK and JNK signaling pathways are shown above. Besides the circulatory regulation of AMPK-SIRT1-LKB1, AMPK indirectly regulates NF- $\kappa \mathrm{B}$ p 65 by SIRT1. Additionally, AMPK might induce the association of PGC- $1 \alpha$. The interaction of NF- $\kappa \mathrm{B}$ and $\mathrm{I} \kappa \mathrm{B}$ might result in nuclear accumulation and activation of NF- $\kappa \mathrm{B}$, leading to IL-10, IL- $1 \beta$, and IL- 6 transcriptions and expressions. Likewise, TNF- $\alpha$ inhibits AMPK via upregulation of protein phosphatase $2 \mathrm{C}$ (PP2C). In the JNK signaling pathway, the main pathway is that TNF- $\alpha$ activates Map4k4 by combining with TNFR1 to inhibit PPAR $\gamma$. Nevertheless, TNF- $\alpha$ activates NF- $\kappa$ B by phosphorylation of JNK1/2. PPAR $\gamma$ might block the interaction of NF- $\kappa$ B and $\mathrm{I} \kappa \mathrm{B}$.

In addition to the function of glucose reduction and obesity alleviation, the Notch signal modulated macrophage polarization. The activation of A disinterring and metalloproteinase (ADAM) domain-type proteinase and $\gamma$-secretase complex were induced by the combination of the Notch receptor with the ligand, DII4 [62]. The Notch intracellular domain (NICD) translocated into the nucleus and bound with the sequence-specific DNA-binding factor RBP-J [55]. These regulations may lead to M1-like polarization. Additionally, the synthesis of interferon regulatory factor 8 (IRF8), a transcription factor, might be promoted by RBP-J via selectively augmenting IRAK2-Mnk1-eif4E axis signaling of TLR4 [62]. TLR4 can both upregulate NF- $\kappa$ B and activate RBP-J. Conversely, SOCS3 plays a crucial role in inhibition of M1-like polarization in the downstream regulation pathway $[55,63]$. The Notch signaling pathway has recently been a focus of research. Thus, the main topic might be whether the Notch could be the mediator and potential treatment strategy in macrophage recruitment or other immune cell interactions in adipose tissue inflammation (Figure 2).

\section{Prospective Compounds for Local Adipose Tissue Chronic Inflammation and Obesity- Induced Insulin Resistance}

Based on the above signaling pathways, prospective compounds have been studied for ameliorating local adipose tissue chronic inflammation with obesity-induced insulin resistance, also known as metaflammation [64]. Bitter melon has been found to ameliorate insulin resistance partly by modulating the inflammatory status. Specifically, it might block NF- $\kappa \mathrm{B}$ by degradation of $\mathrm{I} \kappa \mathrm{B} \alpha$ and suppress phosphorylation of JNK/p38 MAPKs, which were observed not only in epididymal fats but also in the liver and muscle $[65,66]$. For JNK or MAPK signals, benzenediamine derivative FC98 also reduced insulin resistance against metaflammation based on a model of diet-induced obese C57BL/6J mice with the effect of JNK and p38 [67]. Another potential flavonol, (-)-epicatechin, prevented TNF- $\alpha$-triggered activation of inflammation and insulin resistance via suppressing JNK or ERK phosphorylation [68].

More prospective compounds might also interact with proteins in the AMPK signaling pathway. It has been reported that brown alga Ecklonia cava polyphenol extract or quercetin with antioxidative or anti-inflammatory activities modulated AMPK and SIRT1 by reducing adipose tissue mass and lipid accumulation or attenuating macrophage recruitment in epididymis adipose tissue $[69,70]$. In detail, quercetin as flavonol-attenuated adipogenesis in 3T3-L1 cells activated the expression of AMPK or decreased the levels of phosphorylated ERK and JNK and inhibited PPAR $\gamma$ and enhancer-binding protein $\alpha(\mathrm{C} / \mathrm{EBP} \alpha)$ in mRNA and protein expressions $[71,72]$. It was studied in primary bone marrowderived macrophages or macrophage cell lines (human U937 monocytes, murine J774 macrophages) and human 


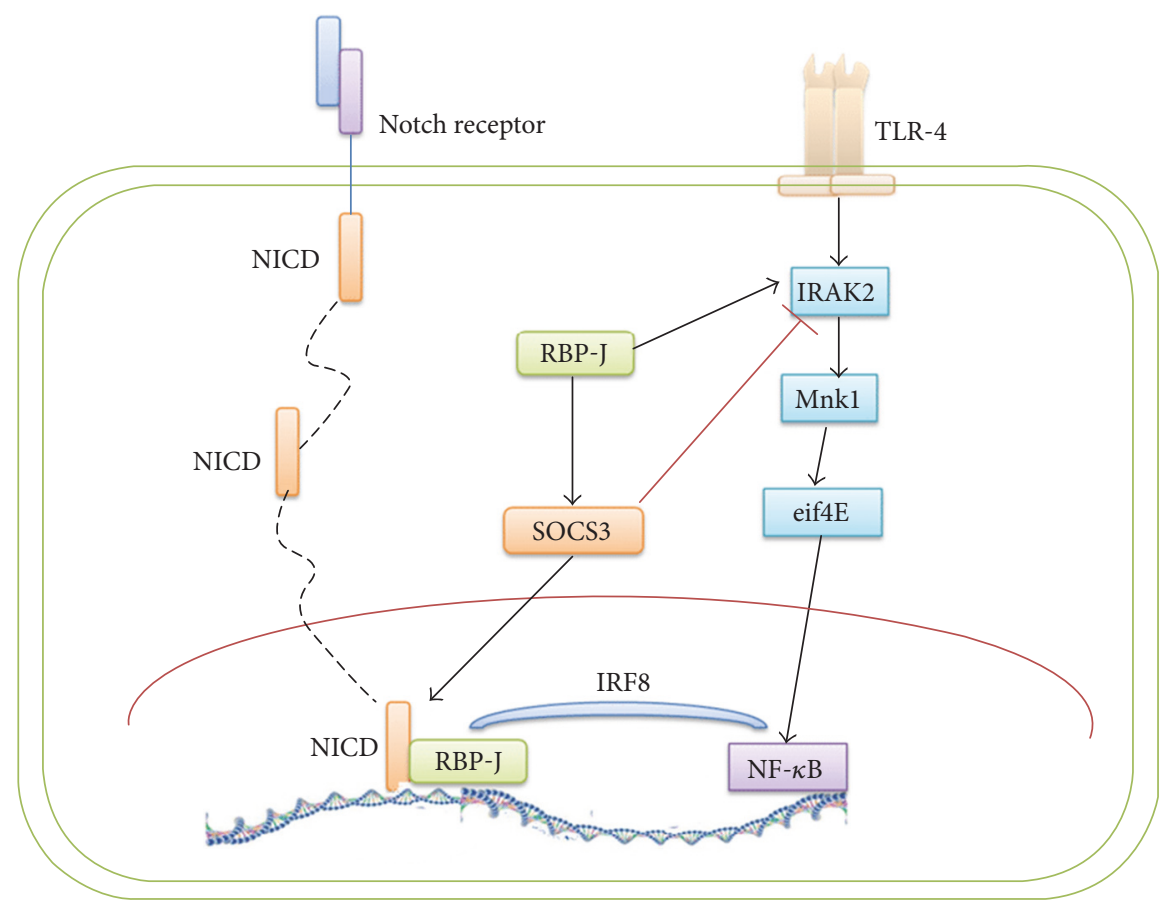

FIgURE 2: The Notch signaling pathways are shown mainly above. The Notch receptor on the cell membrane combines with a DII4 ligand and induces the translocation of NICD from cytoplasm into cell nucleus, binding with RNP-J. Likewise, TLR4 could play a role in Notch signaling pathway to regulate IRAK2, eif4E, and NF- $\kappa$ B, activating the RBP-J signal. However, SOCS3 inhibits one key protein IRAK2 among the signals above.

adipocytes to prove that quercetin possessed the functions of anti-inflammation in local adipose tissue. Quercetin decreased the secretion of TNF- $\alpha$, IL- 6 , and IL- $1 \beta$ and increased IL-10 production. Additionally, it inhibited NO production and iNOS expression by blocking NF- $\kappa \mathrm{B}$, the transcription factor of iNOS [73-75]. Therefore, this evidence explains anti-inflammatory activity of flavonoid quercetin. Similarly, berberine, the extract of rhizoma coptidis, has been studied for several years regarding the activation of AMPK with metabolic effects. It has been used and studied for its properties of reducing body weight, plasma triglycerides, and some inflammatory cytokines or chemokines [76, 77]. As a derivative of berberine, nandinine has also been reported to have an activation effect in AMPK and blocking $\mathrm{I} \kappa \mathrm{B}-\beta$ activation for attenuating insulin resistance in adipocytes [78]. Additionally, berberine strongly suppressed MCP-1 production in macrophages and affected the level of leptin and the expression of PPAR $\gamma$ with downregulation of TNF- $\alpha$, IL-6, and so forth $[79,80]$. The alkaloid berberine, as a traditional Chinese medicine, could regulate AMPK directly and suppress adipokine and inflammatory cytokine production by affecting protein expression in the inflammation pathway, which might be a prospective drug for ameliorating obesity-associated chronic inflammation and improving insulin sensitivity. Both the structural analysis and the pharmacological mechanism should be studied further. However, all the regulating approaches above might provide some thoughts and targets for drug development about adipose tissue-specialized immunologic features or relevant diseases.

\section{Conclusion}

Generally, as the number of obese people increases, a variety of obesity-related diseases and low-grade chronic inflammation cause injury in people's lives and health to different extents. These relative complications might obviously influence the health and quality of daily life. For obesity-induced inflammation, the possibility of macrophage accumulation has been concluded to have three aspects: macrophage recruitment or infiltration, macrophage polarization, and macrophage-like phenotype preadipocytes. All these aspects shed light on the functional plasticity of macrophages and provide potential therapies to regulate of obesity-induced chronic inflammation. Moreover, AMPK, JNK, and Notch signaling should be the critical pathways for regulating macrophage and adipose tissue local inflammation. These pathways are associated with PPAR $\gamma$ or NF- $\kappa \mathrm{B}$ to some extent. Therefore, the aim of the research on the obesity-induced local chronic inflammation mechanism might be focused on the mediators PPAR $\gamma$ or NF- $\kappa \mathrm{B}$, potentially the key points for regulating inflammation. The relationships between PPAR $\gamma$ and NF- $\kappa$ B or among PPAR $\gamma$, NF- $\kappa \mathrm{B}$, and other relevant pathways should also be discussed. It could be another interesting debate whether there is a factor or molecule that could inhibit or activate these mediators and classical signaling pathways to become biomarkers in the treatment of local chronic inflammation, insulin resistance, and type 2 diabetes. Because berberine could modulate AMPK for improving insulin sensitivity, how berberine regulates AMPK and downstream proteins for anti-inflammation in local adipose 
tissue should be elucidated in detail. Similarly, adipose tissue as an immune organ might explain the pathogenesis of local insulin resistance and systemic insulin resistance induced by obesity in a local inflammatory method or low-grade inflammation. These discoveries could provide novel therapies or detection strategies for obesity-related inflammation and complications.

\section{Conflicts of Interest}

The authors declare that they have no conflicts of interest

\section{Acknowledgments}

This work was supported by the National Natural Science Foundation of China (no. 81570762). This work was also supported by the Norman Bethune B Program of Jilin University (2015224), Young Scholars Program of Norman Bethune Health Science Center of Jilin University (2013201011), and Jilin Science \& Technology Development Plan (20150101156JC, 20140203011YY, 20140414052GH, and 20142045). And this work was also conducted in the Key Lab of Traditional Medicine for Diabetes of Jilin Province and Preclinical Pharmacology R\&D Center of Jilin Province.

\section{References}

[1] A. W. Ferrante Jr., "Obesity-induced inflammation: a metabolic dialogue in the language of inflammation," Journal of Internal Medicine, vol. 262, no. 4, pp. 408-414, 2007.

[2] A. S. Greenberg and M. S. Obin, "Obesity and the role of adipose tissue in inflammation and metabolism," The American Journal of Clinical Nutrition, vol. 83, no. 2, pp. 461S-465S, 2006.

[3] M. A. McArdle, O. M. Finucane, R. M. Connaughton, A. M. McMorrow, and H. M. Roche, "Mechanisms of obesity-induced inflammation and insulin resistance: insights into the emerging role of nutritional strategies," Frontiers in Endocrinology, vol. 4, p. 52, 2013.

[4] S. Tateya, F. Kim, and Y. Tamori, "Recent advances in obesityinduced inflammation and insulin resistance," Frontiers in Endocrinology, vol. 4, p. 93, 2013.

[5] P. Manna and S. K. Jain, "Obesity, oxidative stress, adipose tissue dysfunction, and the associated health risks: causes and therapeutic strategies," Metabolic Syndrome and Related Disorders, vol. 13, no. 10, pp. 423-444, 2015.

[6] M. I. Cybulsky, C. Cheong, and C. S. Robbins, "Macrophages and dendritic cells: partners in atherogenesis," Circulation Research, vol. 118, no. 4, pp. 637-652, 2016.

[7] G. Hoeffel, J. Chen, Y. Lavin et al., "C-Myb(+) erythro-myeloid progenitor-derived fetal monocytes give rise to adult tissueresident macrophages," Immunity, vol. 42, no. 4, pp. 665678, 2015.

[8] S. Epelman, K. J. Lavine, A. E. Beaudin et al., "Embryonic and adult-derived resident cardiac macrophages are maintained through distinct mechanisms at steady state and during inflammation," Immunity, vol. 40, no. 1, pp. 91-104, 2014.

[9] C. Schneider, S. P. Nobs, M. Kurrer, H. Rehrauer, C. Thiele, and M. Kopf, "Induction of the nuclear receptor PPARgamma by the cytokine GM-CSF is critical for the differentiation of fetal monocytes into alveolar macrophages," Nature Immunology, vol. 15, no. 11, pp. 1026-1037, 2014.

[10] C. Schulz, E. Gomez Perdiguero, L. Chorro et al., "A lineage of myeloid cells independent of Myb and hematopoietic stem cells," Science, vol. 336, no. 6077, pp. 86-90, 2012.

[11] C. Auffray, M. H. Sieweke, and F. Geissmann, "Blood monocytes: development, heterogeneity, and relationship with dendritic cells," Annual Review of Immunology, vol. 27, pp. 669-692, 2009.

[12] D. K. Fogg, C. Sibon, C. Miled et al., "A clonogenic bone marrow progenitor specific for macrophages and dendritic cells," Science, vol. 311, no. 5757, pp. 83-87, 2006.

[13] H. Iwasaki and K. Akashi, "Myeloid lineage commitment from the hematopoietic stem cell," Immunity, vol. 26, no. 6, pp. 726740, 2007.

[14] M. A. Bouhlel, B. Derudas, E. Rigamonti et al., "PPARgamma activation primes human monocytes into alternative M2 macrophages with anti-inflammatory properties," Cell Metabolism, vol. 6, no. 2, pp. 137-143, 2007.

[15] G. Charriere, B. Cousin, E. Arnaud et al., "Preadipocyte conversion to macrophage. Evidence of plasticity," The Journal of Biological Chemistry, vol. 278, no. 11, pp. 9850-9855, 2003.

[16] S. P. Weisberg, D. McCann, M. Desai, M. Rosenbaum, R. L. Leibel, and A. W. Ferrante Jr., "Obesity is associated with macrophage accumulation in adipose tissue," The Journal of Clinical Investigation, vol. 112, no. 12, pp. 1796-1808, 2003.

[17] H. Xu, G. T. Barnes, Q. Yang et al., "Chronic inflammation in fat plays a crucial role in the development of obesity-related insulin resistance," The Journal of Clinical Investigation, vol. 112, no. 12, pp. 1821-1830, 2003.

[18] C. N. Lumeng, J. L. Bodzin, and A. R. Saltiel, "Obesity induces a phenotypic switch in adipose tissue macrophage polarization," The Journal of Clinical Investigation, vol. 117, no. 1, pp. 175-184, 2007.

[19] Y. H. Lee, S. Nair, E. Rousseau et al., "Microarray profiling of isolated abdominal subcutaneous adipocytes from obese vs non-obese Pima Indians: increased expression of inflammation-related genes," Diabetologia, vol. 48, no. 9, pp. 1776-1783, 2005.

[20] K. L. Spalding, E. Arner, P. O. Westermark et al., "Dynamics of fat cell turnover in humans," Nature, vol. 453, no. 7196, pp. 783-787, 2008.

[21] C. K. Mueller and S. Schultze-Mosgau, "Histomorphometric analysis of the phenotypical differentiation of recruited macrophages following subcutaneous implantation of an allogenous acellular dermal matrix," International Journal of Oral and Maxillofacial Surgery, vol. 40, no. 4, pp. 401-407, 2011.

[22] K. Fujiu, I. Manabe, and R. Nagai, "Renal collecting duct epithelial cells regulate inflammation in tubulointerstitial damage in mice," The Journal of Clinical Investigation, vol. 121, no. 9, pp. 3425-3441, 2011.

[23] T. A. Wynn, L. Barron, R. W. Thompson et al., "Quantitative assessment of macrophage functions in repair and fibrosis," Current Protocols in Immunology, vol. Chapter 14, pp. Unit14-Unit22, 2011.

[24] A. Coste, M. Dubourdeau, M. D. Linas et al., "PPARgamma promotes mannose receptor gene expression in murine macrophages and contributes to the induction of this receptor by IL-13," Immunity, vol. 19, no. 3, pp. 329-339, 2003.

[25] A. Gales, A. Conduche, J. Bernad et al., "PPARgamma controls Dectin-1 expression required for host antifungal defense 
against Candida albicans," PLoS Pathogens, vol. 6, no. 1, article e1000714, 2010.

[26] O. M. Finucane, C. M. Reynolds, F. C. McGillicuddy, and H. M. Roche, "Insights into the role of macrophage migration inhibitory factor in obesity and insulin resistance," The Proceedings of the Nutrition Society, vol. 71, no. 4, pp. 622633, 2012.

[27] M. E. Cabalen, M. F. Cabral, L. M. Sanmarco et al., "Chronic Trypanosoma cruzi infection potentiates adipose tissue macrophage polarization toward an anti-inflammatory M2 phenotype and contributes to diabetes progression in a diet-induced obesity model," Oncotarget, vol. 7, no. 12, pp. 13400-13415, 2016.

[28] W. Ying, S. Kanameni, C. A. Chang et al., "Interferon tau alleviates obesity-induced adipose tissue inflammation and insulin resistance by regulating macrophage polarization," PloS One, vol. 9, no. 6, article e98835, 2014.

[29] M. Zhang, Z. Zhou, J. Wang, and S. Li, "MiR-130b promotes obesity associated adipose tissue inflammation and insulin resistance in diabetes mice through alleviating M2 macrophage polarization via repression of PPAR-gamma," Immunology Letters, vol. 180, pp. 1-8, 2016.

[30] J. Wu, P. Bostrom, L. M. Sparks et al., "Beige adipocytes are a distinct type of thermogenic fat cell in mouse and human," Cell, vol. 150, no. 2, pp. 366-376, 2012.

[31] V. Peirce, S. Carobbio, and A. Vidal-Puig, "The different shades of fat," Nature, vol. 510, no. 7503, pp. 76-83, 2014.

[32] J. Sanchez-Gurmaches and D. A. Guertin, "Adipocyte lineages: tracing back the origins of fat," Biochimica et Biophysica Acta, vol. 1842, no. 3, pp. 340-351, 2014.

[33] J. Sanchez-Gurmaches, C. M. Hung, C. A. Sparks, Y. Tang, H. Li, and D. A. Guertin, "PTEN loss in the Myf5 lineage redistributes body fat and reveals subsets of white adipocytes that arise from Myf5 precursors," Cell Metabolism, vol. 16, no. 3, pp. 348-362, 2012.

[34] J. A. Timmons, K. Wennmalm, O. Larsson et al., "Myogenic gene expression signature establishes that brown and white adipocytes originate from distinct cell lineages," Proceedings of the National Academy of Sciences of the United States of America, vol. 104, no. 11, pp. 4401-4406, 2007.

[35] B. Cousin, O. Munoz, M. Andre et al., "A role for preadipocytes as macrophage-like cells," FASEB Journal, vol. 13, no. 2, pp. 305-312, 1999.

[36] A. Giordano, A. Smorlesi, A. Frontini, G. Barbatelli, and S. Cinti, "White, brown and pink adipocytes: the extraordinary plasticity of the adipose organ," European Journal of Endocrinology, vol. 170, no. 5, pp. R159-R171, 2014.

[37] P. Isakson, A. Hammarstedt, B. Gustafson, and U. Smith, "Impaired preadipocyte differentiation in human abdominal obesity: role of Wnt, tumor necrosis factor-alpha, and inflammation," Diabetes, vol. 58, no. 7, pp. 1550-1557, 2009.

[38] Y. Minokoshi, T. Alquier, N. Furukawa et al., "AMP-kinase regulates food intake by responding to hormonal and nutrient signals in the hypothalamus," Nature, vol. 428, no. 6982, pp. 569-574, 2004.

[39] S. Park, S. Kim da, S. Kang, and B. K. Shin, "Chronic activation of central AMPK attenuates glucose-stimulated insulin secretion and exacerbates hepatic insulin resistance in diabetic rats," Brain Research Bulletin, vol. 108, pp. 18-26, 2014.

[40] R. Stark, S. E. Ashley, and Z. B. Andrews, "AMPK and the neuroendocrine regulation of appetite and energy expenditure,"
Molecular and Cellular Endocrinology, vol. 366, no. 2, pp. 215-223, 2013.

[41] M. B. Marques, A. Ribeiro-Oliveira Jr., J. Guimaraes et al., "Modifications in basal and stress-induced hypothalamic AMP-activated protein kinase (AMPK) activity in rats chronically treated with an angiotensin II receptor blocker," Stress, vol. 15, no. 5, pp. 554-561, 2012.

[42] T. Sanli, G. R. Steinberg, G. Singh, and T. Tsakiridis, "AMPactivated protein kinase (AMPK) beyond metabolism: a novel genomic stress sensor participating in the DNA damage response pathway," Cancer Biology \& Therapy, vol. 15, no. 2, pp. 156-169, 2014.

[43] A. Salminen, J. M. Hyttinen, and K. Kaarniranta, "AMPactivated protein kinase inhibits NF-kappaB signaling and inflammation: impact on healthspan and lifespan," Journal of Molecular Medicine, vol. 89, no. 7, pp. 667-676, 2011.

[44] S. Hummasti and G. S. Hotamisligil, "Endoplasmic reticulum stress and inflammation in obesity and diabetes," Circulation Research, vol. 107, no. 5, pp. 579-591, 2010.

[45] E. Maury and S. M. Brichard, "Adipokine dysregulation, adipose tissue inflammation and metabolic syndrome," Molecular and Cellular Endocrinology, vol. 314, no. 1, pp. 1-16, 2010.

[46] A. Bai, A. G. Ma, M. Yong et al., "AMPK agonist downregulates innate and adaptive immune responses in TNBSinduced murine acute and relapsing colitis," Biochemical Pharmacology, vol. 80, no. 11, pp. 1708-1717, 2010.

[47] G. R. Steinberg, B. J. Michell, B. J. van Denderen et al., "Tumor necrosis factor alpha-induced skeletal muscle insulin resistance involves suppression of AMP-kinase signaling," Cell Metabolism, vol. 4, no. 6, pp. 465-474, 2006.

[48] Y. Sun, J. Li, N. Xiao et al., "Pharmacological activation of AMPK ameliorates perivascular adipose/endothelial dysfunction in a manner interdependent on AMPK and SIRT1," Pharmacological Research, vol. 89, pp. 19-28, 2014.

[49] C. Canto, Z. Gerhart-Hines, J. N. Feige et al., "AMPK regulates energy expenditure by modulating $\mathrm{NAD}+$ metabolism and SIRT1 activity," Nature, vol. 458, no. 7241, pp. 1056-1060, 2009.

[50] F. Lan, J. M. Cacicedo, N. Ruderman, and Y. Ido, "SIRT1 modulation of the acetylation status, cytosolic localization, and activity of LKB1. Possible role in AMP-activated protein kinase activation," The Journal of Biological Chemistry, vol. 283, no. 41, pp. 27628-27635, 2008.

[51] F. Yeung, J. E. Hoberg, C. S. Ramsey et al., "Modulation of NFkappaB-dependent transcription and cell survival by the SIRT1 deacetylase," The EMBO Journal, vol. 23, no. 12, pp. 2369-2380, 2004.

[52] J. Morari, A. S. Torsoni, G. F. Anhe et al., "The role of proliferator-activated receptor gamma coactivator-1alpha in the fatty-acid-dependent transcriptional control of interleukin10 in hepatic cells of rodents," Metabolism, vol. 59, no. 2, pp. 215-223, 2010.

[53] D. N. Dhanasekaran and E. P. Reddy, "JNK signaling in apoptosis," Oncogene, vol. 27, no. 48, pp. 6245-6251, 2008.

[54] L. W. Wang, Y. F. Tu, C. C. Huang, and C. J. Ho, "JNK signaling is the shared pathway linking neuroinflammation, bloodbrain barrier disruption, and oligodendroglial apoptosis in the white matter injury of the immature brain," Journal of Neuroinflammation, vol. 9, no. 1, p. 175, 2012.

[55] D. Zhou, C. Huang, Z. Lin et al., "Macrophage polarization and function with emphasis on the evolving roles of 
coordinated regulation of cellular signaling pathways," Cellular Signalling, vol. 26, no. 2, pp. 192-197, 2014.

[56] R. J. Davis, "Signal transduction by the JNK group of MAP kinases," Cell, vol. 103, no. 2, pp. 239-252, 2000.

[57] H. You, R. M. Padmashali, A. Ranganathan et al., "JNK regulates compliance-induced adherens junctions formation in epithelial cells and tissues," Journal of Cell Science, vol. 126, no. 12, pp. 2718-2729, 2013.

[58] M. S. Han, D. Y. Jung, C. Morel et al., "JNK expression by macrophages promotes obesity-induced insulin resistance and inflammation," Science, vol. 339, no. 6116, pp. 218-222, 2013.

[59] G. J. Tesz, A. Guilherme, K. V. Guntur et al., "Tumor necrosis factor alpha (TNFalpha) stimulates Map4k4 expression through TNFalpha receptor 1 signaling to c-Jun and activating transcription factor 2," The Journal of Biological Chemistry, vol. 282, no. 27, pp. 19302-19312, 2007.

[60] R. Kopan and M. X. Ilagan, "The canonical Notch signaling pathway: unfolding the activation mechanism," Cell, vol. 137, no. 2, pp. 216-233, 2009.

[61] P. Bi, T. Shan, W. Liu et al., "Inhibition of Notch signaling promotes browning of white adipose tissue and ameliorates obesity," Nature Medicine, vol. 20, no. 8, pp. 911-918, 2014.

[62] H. Xu, J. Zhu, S. Smith et al., "Notch-RBP-J signaling regulates the transcription factor IRF8 to promote inflammatory macrophage polarization," Nature Immunology, vol. 13, no. 7, pp. 642-650, 2012.

[63] Y. C. Wang, F. He, F. Feng et al., "Notch signaling determines the M1 versus M2 polarization of macrophages in antitumor immune responses," Cancer Research, vol. 70, no. 12, pp. 4840-4849, 2010.

[64] M. F. Gregor and G. S. Hotamisligil, "Inflammatory mechanisms in obesity," Annual Review of Immunology, vol. 29, pp. 415-445, 2011.

[65] J. Bai, Y. Zhu, and Y. Dong, "Response of gut microbiota and inflammatory status to bitter melon (Momordica charantia L.) in high fat diet induced obese rats," Journal of Ethnopharmacology, vol. 194, pp. 717-726, 2016.

[66] S. J. Yang, J. M. Choi, S. E. Park et al., "Preventive effects of bitter melon (Momordica charantia) against insulin resistance and diabetes are associated with the inhibition of NF-kappaB and JNK pathways in high-fat-fed OLETF rats," The Journal of Nutritional Biochemistry, vol. 26, no. 3, pp. 234-240, 2015.

[67] C. Chen, W. Zhang, H. Shi et al., "A novel benzenediamine derivative FC98 reduces insulin resistance in high fat dietinduced obese mice by suppression of metaflammation," European Journal of Pharmacology, vol. 761, pp. 298-308, 2015.

[68] M. A. Vazquez-Prieto, A. Bettaieb, F. G. Haj, C. G. Fraga, and P. I. Oteiza, "(-)-Epicatechin prevents TNFalpha-induced activation of signaling cascades involved in inflammation and insulin sensitivity in 3T3-L1 adipocytes," Archives of Biochemistry and Biophysics, vol. 527, no. 2, pp. 113-118, 2012.

[69] J. Dong, X. Zhang, L. Zhang et al., "Quercetin reduces obesityassociated ATM infiltration and inflammation in mice: a mechanism including AMPKalpha1/SIRT1," Journal of Lipid Research, vol. 55, no. 3, pp. 363-374, 2014.

[70] H. Eo, Y. J. Jeon, M. Lee, and Y. Lim, "Brown alga Ecklonia cava polyphenol extract ameliorates hepatic lipogenesis, oxidative stress, and inflammation by activation of AMPK and SIRT1 in high-fat diet-induced obese mice," Journal of Agricultural and Food Chemistry, vol. 63, no. 1, pp. 349-359, 2015.
[71] J. Ahn, H. Lee, S. Kim, J. Park, and T. Ha, "The anti-obesity effect of quercetin is mediated by the AMPK and MAPK signaling pathways," Biochemical and Biophysical Research Communications, vol. 373, no. 4, pp. 545-549, 2008.

[72] L. Yang, X. F. Li, L. Gao, Y. O. Zhang, and G. P. Cai, "Suppressive effects of quercetin-3-O-(6"-feruloyl)-beta-D-galactopyranoside on adipogenesis in 3T3-L1 preadipocytes through downregulation of PPARgamma and C/EBPalpha expression," Phytotherapy Research, vol. 26, no. 3, pp. 438-444, 2012.

[73] M. Comalada, I. Ballester, E. Bailon et al., "Inhibition of proinflammatory markers in primary bone marrow-derived mouse macrophages by naturally occurring flavonoids: analysis of the structure-activity relationship," Biochemical Pharmacology, vol. 72, no. 8, pp. 1010-1021, 2006.

[74] M. Hamalainen, R. Nieminen, P. Vuorela, M. Heinonen, and E. Moilanen, "Anti-inflammatory effects of flavonoids: genistein, kaempferol, quercetin, and daidzein inhibit STAT-1 and NF-kappaB activations, whereas flavone, isorhamnetin, naringenin, and pelargonidin inhibit only NF-kappaB activation along with their inhibitory effect on iNOS expression and NO production in activated macrophages," Mediators of Inflammation, vol. 2007, Article ID 45673, p. 10, 2007.

[75] A. Overman, C. C. Chuang, and M. McIntosh, "Quercetin attenuates inflammation in human macrophages and adipocytes exposed to macrophage-conditioned media," International Journal of Obesity, vol. 35, no. 9, pp. 1165-1172, 2011.

[76] Y. S. Lee, W. S. Kim, K. H. Kim et al., "Berberine, a natural plant product, activates AMP-activated protein kinase with beneficial metabolic effects in diabetic and insulin-resistant states," Diabetes, vol. 55, no. 8, pp. 2256-2264, 2006.

[77] X. Zhang, Y. Zhao, M. Zhang et al., "Structural changes of gut microbiota during berberine-mediated prevention of obesity and insulin resistance in high-fat diet-fed rats," PloS One, vol. 7, no. 8, article e42529, 2012.

[78] W. Zhao, H. Ge, K. Liu, X. Chen, J. Zhang, and B. Liu, "Nandinine, a derivative of berberine, inhibits inflammation and reduces insulin resistance in adipocytes via regulation of AMP-kinase activity," Planta Medica, vol. 83, no. 3-4, pp. 203-209, 2017.

[79] B. H. Choi, I. S. Ahn, Y. H. Kim et al., "Berberine reduces the expression of adipogenic enzymes and inflammatory molecules of 3T3-L1 adipocyte," Experimental \& Molecular Medicine, vol. 38, no. 6, pp. 599-605, 2006.

[80] A. Remppis, F. Bea, H. J. Greten et al., "Rhizoma coptidis inhibits LPS-induced MCP-1/CCL2 production in murine macrophages via an AP-1 and NFkappaB-dependent pathway," Mediators of Inflammation, vol. 2010, Article ID 194896, p. 8, 2010. 


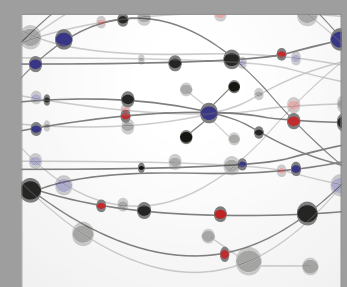

The Scientific World Journal
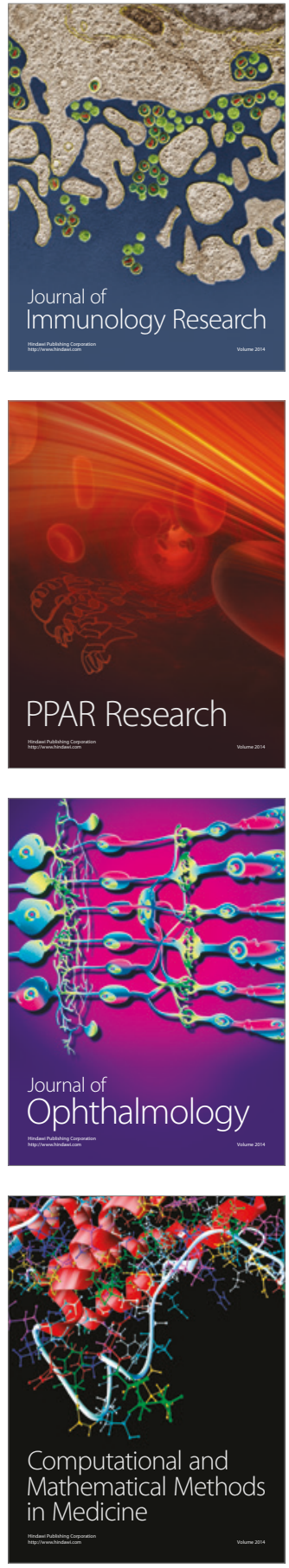

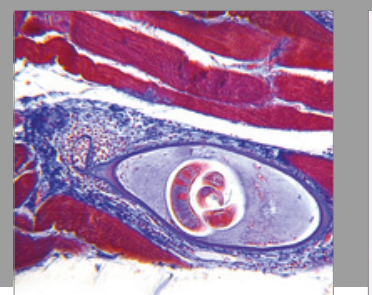

Gastroenterology Research and Practice
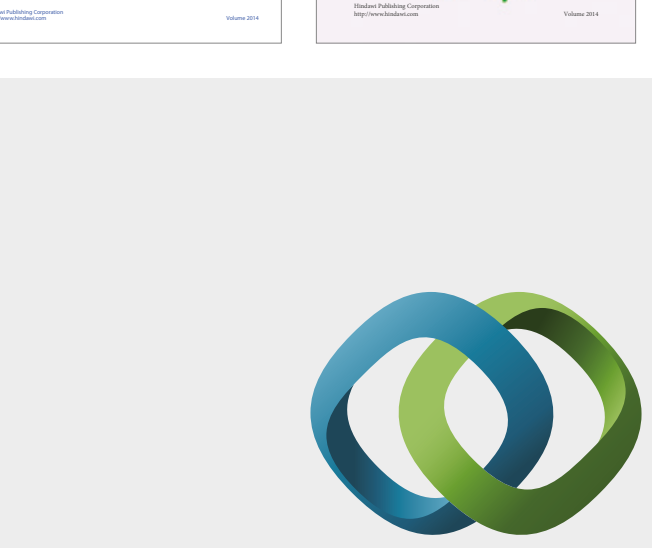

\section{Hindawi}

Submit your manuscripts at

https://www.hindawi.com
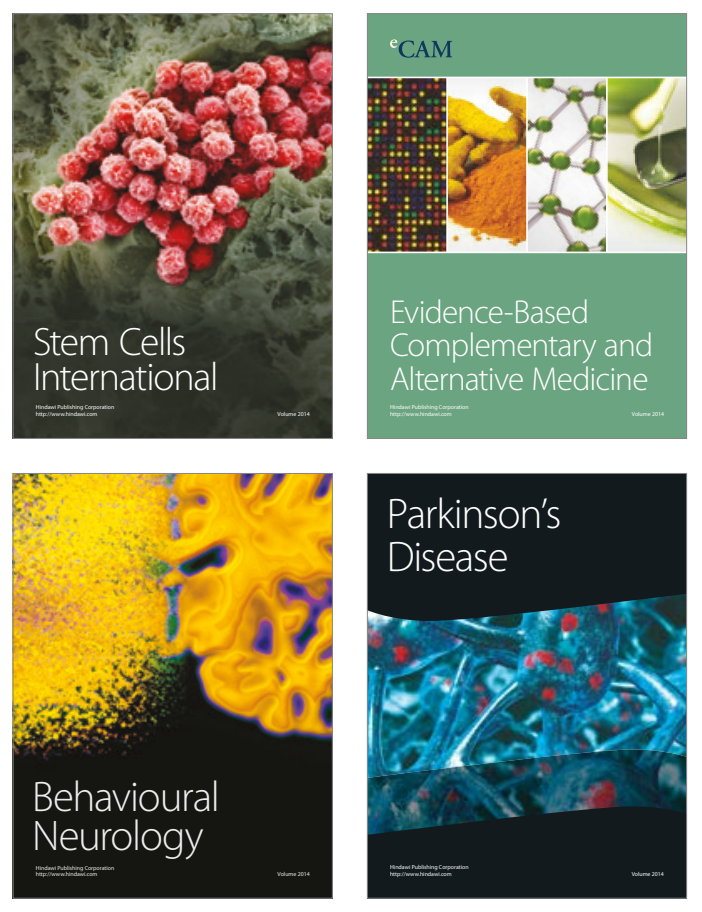
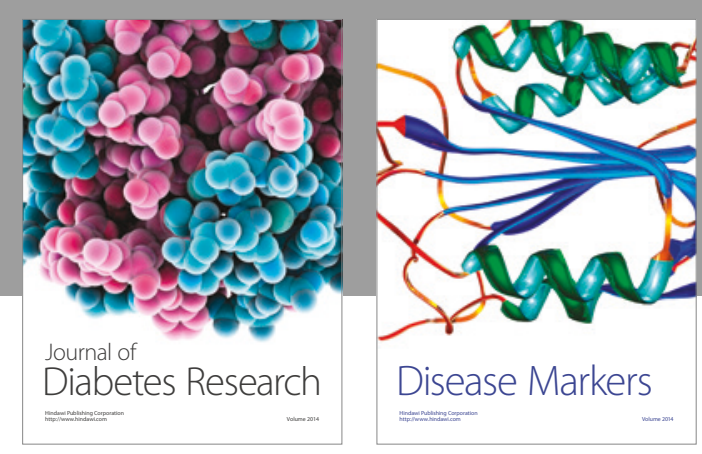

Disease Markers
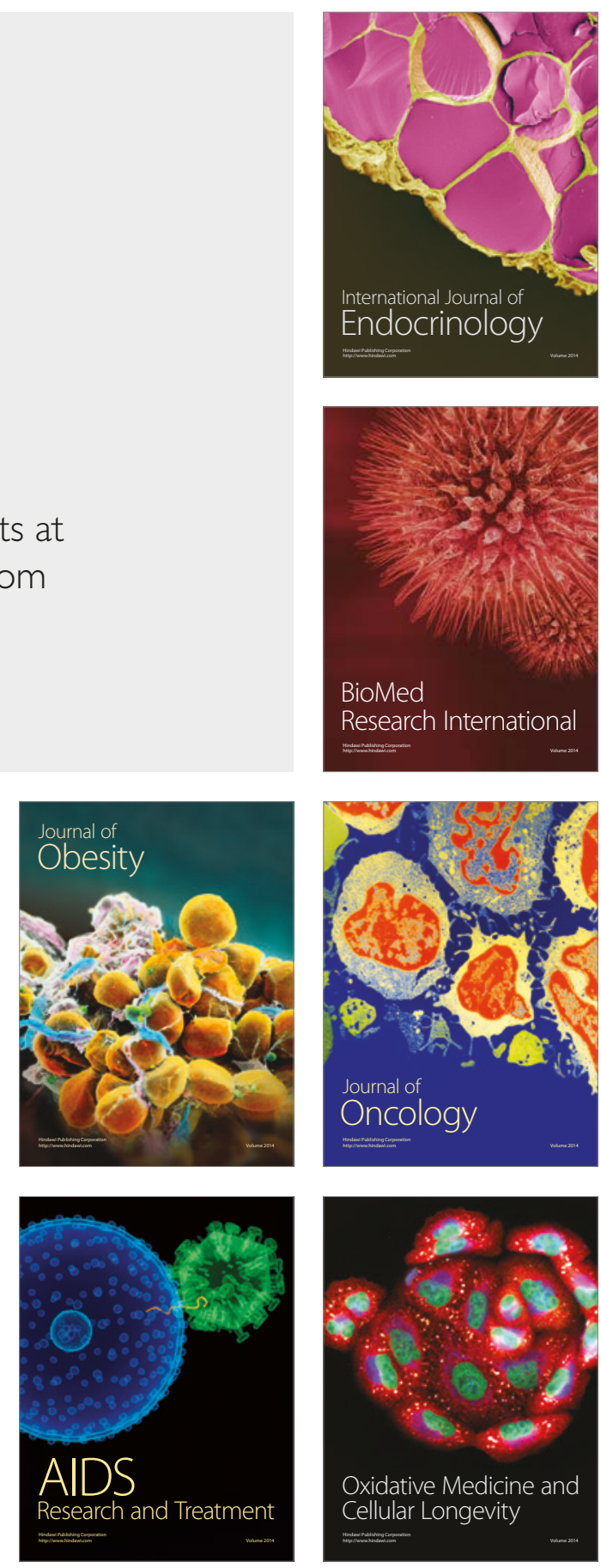